\section{Patient information on chemotherapy-induced bone marrow suppression}

\section{By Joan Hamilton, Leslie McLean and Eileen Patterson}

\section{Abstract}

Given the few resources available to complement patient teaching on the subject of chemotherapy-induced myelosuppression, the following information sheets were developed for patients receiving chemotherapy: "What Are My Counts?", "Low Red Blood Cell Count", "Low White Blood Cell Count" and "Low Platelet Count". These resources are designed for patients who experience moderate to profound bone marrow suppression.

Although much has been written for the nurse about chemotherapy-induced myelosuppression, there are very few nursing resources available to complement patient teaching on the subject. In response to this need, the following information sheets were developed for the patient receiving myelosuppressive chemotherapy.

The degree of myelosuppression a patient experiences as a side effect of chemotherapy depends on a number of variables relating to both the patient and the cytotoxic drug (i.e., the type of chemotherapeutic agent, the dose and frequency at which it is administered, the patient's age and disease status). These particular information sheets have been designed for patients who experience moderate to profound bone marrow suppression. It is recommended that the nurse using these resources individualize his/her teaching by deleting or changing the content as appropriate to their agency practice and the degree of myelosuppression experienced by the patient.

The effects of chemotherapy on the blood forming cells of the bone marrow, although temporary, can be life-threatening and pose a serious challenge to patients and to the nurses caring for these patients. It is hoped that these resource sheets will assist the nurse in providing the patient with the understanding necessary to withstand

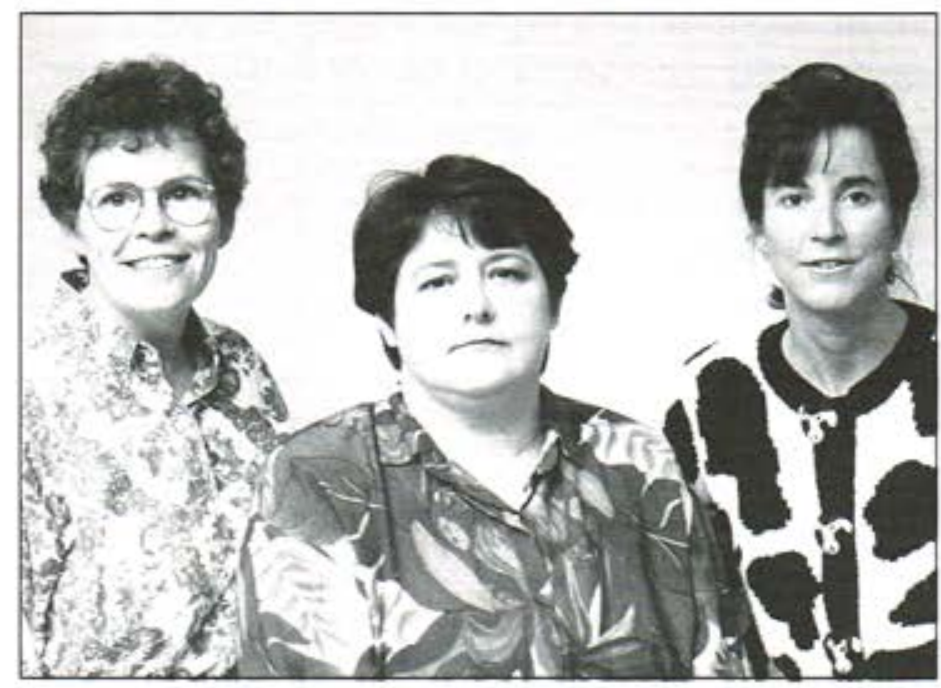

Joan Hamilton, Eileen Patterson and Leslie McLean. these potentially devastating complications.

Using the SMOG readability formula, a patient should have a grade 10 reading level in order to fully understand these information sheets.

\section{Suggested Readings}

The volume of information in the literature related to this subject is extensive. The authors have therefore provided the following selections:

Alkine and Collingwood. (1990). Physiology of blood and bone marrow, Seminars in Oncology Nursing, 6(2), 99-108.

Brown, S. (1990). Behind the numbers on the CBC. RN, Feb., 46-51.

Greifzu, Radjeski and Winnick. (1990). Oral care is part of cancer care. RN, June, 43-45.

Griffin, J. (1986). Hematology and Immunology. Connecticut: Appleton-Century-Crofts. Chapters 1, 2, 3, 5, 6, 8, 11, 12 and 13. Oniboni, A. (1990) Infection in the Neutropenic Patient. Seminars in Oncology Nursing, 6(1), 50-60.

Rostad, M. (1991). Current strategies for managing myelosuppression in patients with cancer. Oncology Nursing Forum, 18(2), Supplement, 7-14.

Rostad, M. (1990). Management of myelosuppression in the patient with cancer. Oncology Nursing Forum, 17(1), Supplement, Jan/Feb., 4-8.

Skalla, K. and Lacasse, C. (1992). Patient Education on Fatigue. Oncology Nursing Forum, 19(10), 1537-1541.

Weikel, Peterson, Rubinstein, Metzger-Samuels and Overholser. (1989). Incidence of fever following invasive oral interventions in the myelosuppressed cancer patient. Cancer Nursing, 12(5), 265-270.

Workman, Ellerhorst-Ryan and Hargrave-Koertge. (1993). Nursing, Care of the Immunocompromised Patient. Toronto: W.B. Saunders.

The authors and the Canadian Oncology Nursing Journal waive copyright on this material in order that it may be widely utilized. The authors would appreciate being informed of any major revisions or changes.

As with any published reference material, once in use, flaws may be found and with new knowledge, practices may change. Each agency/user of this material has the responsibility to ensure this information is applied appropriately to the population they serve.

\section{What Are My Counts?}

(For people receiving chemotherapy)

Blood is made up of cells and fluid. There are three major types of blood cells in the blood. These blood cells are made in the bone marrow. They are called red blood cells, white blood cells and platelets.

The word "counts" refers to the number of red blood cells, white blood cells and platelets in your body.

A blood count is obtained from a sample of blood taken from your vein. It is then examined in a laboratory and the number of red blood cells, white blood cells and platelets are actually counted.

Counts tell a number of things about your condition, for example, how well you are responding to chemotherapy, if you have an increased risk of infection or bleeding, or if you are anemic.

Red Blood Cells - Red blood cells carry oxygen to all parts of your body. The part of the red blood cell that carries the oxygen is called hemoglobin $(\mathrm{Hgb})$. When your red blood cell (or hemoglobin) count is low you have a condition called anemia which can make you feel tired. It is your hemoglobin that is usually monitored (watched) to see if you have anemia.

Joan Hamilton, RN, MSc(N) is Clinical Nurse Specialist, Leslie McLean, RN, BScN is Unit Resource Nurse, and Eileen Patterson, RN, BN, is former Head Nurse in Haematology/Medical Oncology at Victoria General Hospital, Halifax, Nova Scotia. 
A normal hemoglobin $(\mathrm{Hgb})$ is generally between $115-155$ for women and 130-175 for men. If your hemoglobin falls below 80 or 90 , you may be given a red blood cell transfusion to increase the number of your red blood cells.

Platelets - Platelets (PLT) help to make blood clot (stop bleeding). If you do not have enough platelets you may bruise and bleed very easily.

A normal platelet cell count is $150,000-350,000$.

If your platelet count is below 50,000 you might begin to bruise and bleed more easily; below 20,000 you will be given a platelet transfusion; below 10,000 you will be encouraged to stay in bed to decrease your chances of bleeding.

White Blood Cells - White blood cells help to fight off germs (for example, bacteria \& viruses) that can cause infection. If the number of these cells decreases, you have a greater chance of getting an infection.

A normal white cell count is between 4,500-10,500. Your white count number may go as low as 100 .

There are different types of white blood cells, but most of the time you will hear about neutrophils. Neutrophils are specialized white blood cells that are your first line of defense against many infections. Because the number of neutrophils are so important, they are counted separately. The total count of neutrophils is called an absolute neutrophil count (ANC).

The lower your absolute neutrophil count (ANC), the greater the risk of getting an infection. The normal ANC number is 2,500-6,000.

If your ANC is below 1,000 you have a greater chance of getting an infection. An ANC of 500 or less means you will have to take even greater care to prevent infections.

\section{What affects your "counts"?}

Now that you know what these "counts" are, you are probably wondering what makes them go up and down. There are several things that can affect your counts such as; disease, radiotherapy, chemotherapy, medications, injury and infections. The explanation given here will focus on chemotherapy since you are or will be receiving this form of treatment.

You are (or will be) given anticancer drugs called chemotherapy. Chemotherapy works by destroying the cancer cell's ability to grow or make other cancer cells. Unfortunately these drugs can also destroy certain normal cells in your body. The normal cells most frequently affected by chemotherapy are: a) the cells that grow hair; b) the cells in your mouth, down into your stomach and in your intestine, (your gastrointestinal tract); c) cells of the reproductive system; and, d) the cells inside your bones (bone marrow) that produce red blood cells, white blood cells and platelets.

As the chemotherapy destroys the healthy bone marrow cells, your counts drop.

There are many different types of cancer chemotherapy agents. How much your counts will drop and for how long depends on what chemotherapy drugs you receive and how often you get them. As a general rule, you will see your "counts" drop within 7-10 days after your chemotherapy is given. They will probably stay low for 2-3 weeks, at which time they will begin to recover. Sometimes, if your blood counts are too low, your chemotherapy treatment will be postponed until a later date. Remember: This is a general rule and your case may not follow this exactly.

Frequent blood tests are done in order to monitor the changes in your blood counts.

Some people like to know when and how their counts are changing. The nurse can record them on a sheet for you to keep. Other people do not wish to know about their counts. Let your nurse know whether or not you want the information and if you would like it written down.

If you have concerns or questions about your counts at any time ask your nurse or doctor

Hamilton, McLean and Patterson,

Victoria General Hospital, Halifax, NS, May 1994

\section{Low Red Blood Cell Count}

\section{(Anemia)}

\section{(Patient information for people receiving chemotherapy)}

Red blood cells are produced in the bone marrow which is the soft tissue in the centre of most bones. Red blood cells carry oxygen to all parts of your body. The part of the red blood cell that carries the oxygen is called hemoglobin. When your red blood cell (or hemoglobin) count is low, parts of your body do not get enough oxygen to do their work. This condition is called anemia and can make you feel very tired.

A low red blood cell count can be caused by anything that keeps the bone marrow from doing its job well or by anything that destroys the red blood cells in the blood or bone marrow. One cause is chemotherapy.

A major side effect of chemotherapy is that it temporarily decreases the bone marrow's ability to make red blood cells, white blood cells and platelets. All of these may decrease during your treatment. This information sheet deals with the decrease in the number of your red blood cells. Because there is such a range in how much the red blood cell count will drop, you will have to check with the nurse to find out which of the items described below apply to you.

\section{Chemotherapy and the red blood cell:}

Most cancer cells grow and divide very quickly. Chemotherapy drugs work by acting mainly on quickly dividing cells. Unfortunately, chemotherapy drugs can't tell the difference between cancer cells and normal cells that also divide quickly. Since red blood cells divide quickly, they are also killed by chemotherapy drugs.

With chemotherapy, there is only a temporary decrease in the number of red blood cells. Depending on the type and length of time on chemotherapy, the red blood cell count (hemoglobin) may only drop slightly resulting in no symptoms. If there is a large drop in the red blood cell count (severe anemia), a person may experience a variety of signs and symptoms which may require special nursing and medical care.

Blood tests are taken regularly to monitor your hemoglobin levels. A normal hemoglobin ( $\mathrm{Hgb}$ ) is generally between 115-155 for women and 130-175 for men. If your hemoglobin falls below 80 or 90 , you may be given a red blood cell transfusion.

\section{Some signs and symptoms that can be associated with low red blood cells (anemia)}

\section{Fatigue}

Easily upset

No patience

Mood changes

Weight loss

Lack of appetite

Diarrhea

Constipation

Loss of sexual desire

Fast heart rate

Loss of colour in nail beds

Tingling of fingers and toes

Headache

Dizzy

Swelling

Itching

\section{Suggestions to help you live with anemia}

Safety

Because of the decreased amount of oxygen reaching your arms and legs, you may become more sensitive to things such as cold and/or less sensitive to such things as heat or touch. For example, you 
may feel more chilly than usual or you may bump yourself without being aware of it. For this reason, avoid heating pads and hot water bottles, and be extra careful not to injure yourself.

When you feel dizzy or light-headed, lie flat for a few minutes, change your position slowly.

When waking up, sit at the bedside for a few minutes before getting up.

Ask for assistance when you feel unsteady on your feet. In hospital use your call bell, at home call your doctor if dizziness or fainting is new, or if you are noticeably more tired or short of breath.

\section{Activity}

Rest when you are tired and after activities. Don't overwork!

Conserve your energy. Pace your activities.

Don't fight fatigue, learn to live with it while your counts are low.

Let others do things for you.

Exercise a bit each day.

You may want to plan for sexual activities in the mornings after a good night's sleep when you have more energy.

Gradually resume normal activities when recovering from chemotherapy.

Adjust your lifestyle according to your energy level, for example, decide on the "must do's" and the "can waits".

If you are in bed for a large part of the day, it is important to turn every hour and move your legs and arms even if only a small amount.

Try to maintain your usual sleep times.

When conserving your energy and getting lots of rest you may still feel tired, even when waking up from a full night of sleep.

\section{Nutrition}

In order to make and maintain red blood cells you need iron, folic acid and vitamins in your diet. Try to eat green leafy vegetables and cooked red meats.

Drink plenty of fluids, 2-3 litres per day (8-12 cups) unless you have a heart or kidney problem.

Try to eat well-balanced meals. Dietitians are available to offer suggestions and guidance.

Iron supplements are usually constipating, but should be taken if your doctor prescribed them. Try prunes, prune juice, dried fruit, etc. Discuss with the doctor taking a laxative.

Do mouth care frequently and thoroughly.

\section{Mood swings/personality changes}

Remember that some changes in how you normally feel or act may be associated with anemia and are temporary. You may find you become frustrated easily, that you have a shorter attention span and/or things don't interest you as much as usual. Don't be hard on yourself if you don't have your usual humour or "get up and go". Try to get plenty of sleep and pace, don't push yourself.

\section{Family/friends}

Friends and family need to learn and understand your need for rest and how they can support you. They need to be informed of the mood changes and swings that are often associated with anemia. Although they can never completely understand how you feel, the more they learn, the more they will understand.

If you need help with how to explain what you are experiencing to loved ones and/or how they can help you, ask your nurse for assistance.

Hamilton, McLean and Patterson, Victoria General Hospital, Halifax, NS, May 1994

\section{Low white blood cell count (Immunosuppression)}

(Patient information for people receiving chemotherapy)

White blood cells help to protect your body from infection. They are part of the body's immune system. There are different types of white cells but most of the time you will hear about neutrophils (pronounced New troh fils):

The body protects itself against the constant threat of infection by making a lot of neutrophils. They are your main defense against infections. If your neutrophil count (the number of neutrophils in your blood) is low, there is a greater chance you might develop an infection. Neutropenia is a word used to describe a low neutrophil count. Immunosuppression is another term used when white blood cells are low.

\section{What causes your white cell count to decrease?}

White blood cells are produced in the bone marrow which is the soft tissue in the centre of most bones. Neutropenia or immunosuppression can be caused by anything that keeps the bone marrow from doing its job well - or by anything that destroys the white blood cells in the blood or bone marrow. Diseases such as leukemia can cause it. Sometimes a virus, some poisons or certain drugs may be the cause. A drop in the white cell count occurs frequently with the use of cancer chemotherapy.

Most cancer cells grow and divide very quickly. Chemotherapy drugs work by acting on quickly dividing cells. Unfortunately, chemotherapy can't tell the difference between a cancer cell and some normal cells that also divide quickly. White blood cells such as neutrophils which divide quickly are therefore also killed by the chemotherapy. Remember however, that with chemotherapy, the decrease in your white blood cell count is only temporary. It will probably stay low for two to three weeks then begin to rise.

Because there is such a range in how much the white blood cell count will drop, you will have to check with the nurse to find out which of the items described below apply to you.

\section{What it means to have a low white cell count}

A normal WBC (white blood cell) count is between 4,500-10,500. Frequent blood tests will be done, particularly after you receive cancer chemotherapy, to monitor the changes in your blood counts. The number of neutrophils is especially important and can and will be counted separately. This is referred to as your ANC (absolute neutrophil count). A normal ANC is between 2,500 - 6,000.

If your $\mathrm{ANC}$ is below 1,000 you have a greater chance of getting an infection. An ANC of 500 or less means even greater care will have to be taken to decrease your risk of infection.

If your ANC is very low, your cancer treatment may be postponed until a later date.

\section{What you can do to decrease your risk of infection}

\section{Hygiene:}

- Wash hands frequently especially after using the washroom and before eating.

- Maintain good body hygiene

- use liquid rather than bar soap

- bath every day, gently pat skin dry

- use moisturizer to prevent dry skin

- use cuticle cream remover rather than picking or cutting nail cuticles

- use deodorant rather than antiperspirant (which blocks sweat glands and may promote infection)

- after a bowel movement, clean rectal area thoroughly Women

- use napkins instead of tampons for menstruation

- avoid vaginal douches, bubble bath or bath salts

- clean vaginal/anal area front to back 
Mouth care

- avoid hard tooth brushes or dental floss

- for regular cleaning, rinse mouth thoroughly and frequently (for example, after each meal and before bed)

- for a bad taste in your mouth, don't use commercial mouthwashes because they are very drying - try $1 / 2$ tsp. of baking soda and $1 / 2$ tsp. of salt in an 8 ounce glass of water - antibiotics may be recommended before and after any dental work or cleaning (so always mention chemotherapy treatment to your dentist)

\section{Protection:}

- use clean electric razor rather than a straight or twin blade safety razor to avoid breaks in skin

- prevent constipation to avoid irritation or breaking of skin (for example, use prunes or laxatives). Discuss this with your doctor or nurse

- avoid taking rectal temperatures, avoid using rectal or vaginal suppositories (they can introduce infection into the body or tear skin)

- use a lubricant during intercourse

- use a condom if engaging in anal intercourse

- avoid getting a sunburn

- protect hands from cuts and burns

- wear shoes to prevent cuts

- wear gloves when doing physical work

- don't squeeze or scratch pimples

- if break in skin develops, disinfect wound and cover with a band-aid

- avoid contact with animal excreta (feces, urine)

- avoid contact with stagnant water (flower vases, humidifier, denture cups)

- avoid people with colds, flu or any type of infection or open sore

- visitors will have to wear a mask if they have a cold

- avoid enclosed public areas where there are crowds of people

- avoid vaccinations unless approved by a physician. Avoid people recently vaccinated with live vaccine. This would most likely be infants 12-15 months of age who have received their MMR. Do not visit until six weeks after the needle.

\section{Exercise:}

- exercise every day to keep your lungs working well

- get plenty of rest

- learn to manage your stress - your nurse, doctor or social worker may be able to suggest ways to help you with this

\section{Diet:}

- maintain a well-balanced diet

- make sure your food is well cooked

- drink 2-3 litres ( 3 litres = 12 cups) of fluid a day (unless you have a heart or kidney problem)

\section{Signs and symptoms of an infection}

Most infections occur in the mouth, throat, sinus, lungs and skin,

Notify your doctor or nurse if you notice any of the following:

Sore throat, eye/ear discharge, chills (shaking), nasal congestion, break in skin, burning at anus, loose bowel movements, pain, burning feelings on urination, redness, cough, swelling, sweating (especially at night).

Fever - depending on how low your white count is, you may be instructed to take your temperature on a regular basis (for example, daily) otherwise, take your temperature if you are feeling warm or unwell. If you have a fever, (38 degrees celsius or over) contact your doctor.

If you should develop an infection while your white count is low, antibiotics by an intravenous infusion (IV) will most likely be given.

Hamilton, McLean and Patterson, Victoria General Hospital, Halifax, NS, May 1994

\section{Low platelet count (Thrombocytopenia)}

(Patient information for people receiving chemotherapy)

Platelets are produced in the bone marrow which is the soft tissue in the centre of most bones. Platelets help make blood clot (stop bleeding). When your platelet count goes down you may bleed more easily and for a longer time. If your platelet count is really low, the concern is that you will not have enough platelets to form the clots needed to stop bleeding. A low platelet count is called thrombocytopenia.

A low platelet count can be caused by anything that keeps the bone marrow from doing its job well or by anything that destroys the platelets in the blood or bone marrow. One cause is chemotherapy.

A major side effect of chemotherapy is that it temporarily decreases the bone marrow's ability to make red blood cells, white blood cells and platelets. All of these may decrease during your treatments. This information sheet deals with the decrease in your platelet count. Because there is such a range in how much the platelet count will drop you will have to check with the nurse to determine which of the items described below apply to you.

\section{Chemotherapy and platelets:}

Most cancer cells grow and divide very quickly. Chemotherapy drugs work by acting mainly on quickly dividing cells. Unfortunately, chemotherapy can't tell the difference between a cancer cell and normal cells that also divide quickly. Platelets which divide quickly are also killed by chemotherapy. It is important to remember that with chemotherapy, the decrease in your platelet count is only temporary.

In order to monitor your platelet count, blood tests will be taken regularly. A normal platelet count is $150,000-350,000$. If your platelet level is very low (usually below 20,000 ) platelets may be given by an intravenous infusion (IV) so that your body is able to stop any bleeding that may occur.

\section{Signs and symptoms of low platelets:}

- Bruising easily;

- Small pinpoint red spots called petechiae;

- Blood in urine;

- Easy bleeding of gums;

- Vomit that looks like coffee grounds;

- Blood tinged or black bowel movements; and

- Bleeding from cuts and scratches that won't stop.

\section{What to do when you have a low platelet count}

Safety

Avoid activities that can cause physical injury.

Avoid contact sports (anything in which you may bump into things or be bumped into).

Use only an electric razor for shaving (this applies to women as well).

Do not let anyone give you a needle unless your cancer doctor has said it is okay.

Do not wear clothes that are tight, for example; tight collars, neck ties, slacks that are tight at the thigh or waist. Avoid harsh or coarse fabrics.

Avoid blowing your nose. If absolutely necessary, do so gently keeping both nostrils open. Closing off one nostril increases pressure and may cause bleeding.

Take necessary actions to keep your bowel movements soft. This may be done through diet (for example, prunes) or by medications, (for example, stool softeners). Discuss this with the doctor, nurse or dietitian.

When your bowels move, do not hold your breath and push, as this increases internal body pressures greatly. An increase in internal pressure with a low platelet count may cause bleeding to occur inside your body.

If you must pick something off the floor, bend at the knees NOT at the waist. Bending at the waist increases internal body pressure.

If you need to move yourself up in bed, do so by hitching your 
bottom upward along the bed NOT by pushing with both arms on the bed to lift your bottom up. This is another way of keeping internal body pressures from rising.

Some very common medications, even ones on the drug store shelf, can affect platelet production or function in the body (for example, medications that contain aspirin). Check with your doctor before taking ANY medications.

Each day examine your body for bruises and rashes. Report any changes to the nurse or doctor if you are in hospital or call your doctor if you are at home.

If you cut yourself or if bleeding occurs, apply pressure using aclean cloth for at least 5 minutes. Time yourself and DO NOT PEEK. If the bleeding does not stop after 10 minutes, call the doctor.

Be extra careful using tools, knives, while cooking, baking and ironing.

Always wear shoes to protect your feet and gloves to protect your hands when doing physical work.

\section{Skin and moist tissues}

Use a water soluble lubricant during sexual intercourse. This lubricant can be found at the drug store and states "water-soluble" on the package. It is recommended to open new individual packets each time.

There may be certain sexual practices you need to refrain from during this time. Ask your nurse or doctor.

Women - DO NOT use tampons or douches.

Try and keep your lips and skin lubricated to prevent drying and cracking. (Humidifiers are not recommended because they are a great place for germs). If your home is really dry, try hanging your wet washed clothes around the house to dry.

Nose bleeds - sit up straight, apply firm pressure below the bridge of the nose. Apply ice over the bridge of your nose. If bleeding continues for more than 5 minutes (and you are not in hospital) call your doctor.

DO NOT give yourself enemas or suppositories.

Keep nails short and smooth. Trim nails carefully to avoid cuts.

Try to drink at least 3 litres ( 12 cups) of fluid a day unless you have a heart or kidney problem. This will help keep your skin in good condition and will also help your bowel movements remain soft.

\section{Mouth care}

Eat only soft, non-spicy food. You may wish to check with the dietitian.

Do not allow your lips to become dry and cracked. Apply a lubricant often.

Use only a soft toothbrush or toothettes (these are available at the pharmacy).

DO NOT use dental floss, the possibility of causing the gums to bleed is too great.

It is important that you clean your mouth at least every four hours. If you wake during the night you may want to take the opportunity to clean your mouth at that time.

\section{Nutrition}

In order to make platelets you need PROTEIN in your diet. You should eat high protein, high calorie foods. You may want to check with the dietitian, especially if your appetite is poor.

Ask about seeing a dictitian if you think your diet is not well-balanced.

DO NOT use straws unless truly necessary. They can be irritating to the inside of the mouth and tongue.

\section{When should I call my doctor?}

If you bleed anywhere for more than 5 minutes.

If you notice blood in your vomit, urine, sputum or bowel movements.

Blood in a bowel movement may be red, black or tarry coloured so if this is new call your family physician.

If you develop a rash, spots or increased bruises that are new, call your family physician.

Hamilton, McLean and Patterson,

Victoria General Hospital, Halifax, NS, May 1994 